\title{
Wireless Multimedia Communications and Networking Based on JPEG 2000
}

\author{
Max AGUEH \\ ECE Paris
}

France

\section{Introduction}

Nowadays, more and more multimedia applications integrate wireless transmission functionalities. Wireless networks are suitable for those types of applications, due to their ease of deployment and because they yield tremendous advantages in terms of mobility of User Equipment (UE). However, wireless networks are subject to a high level of transmission errors because they rely on radio waves whose characteristics are highly dependent of the transmission environment.

In wireless video transmission applications like the one considered in this chapter and presented in Figure 1, effective data protection is a crucial issue.

JPEG 2000, the newest image representation standard, addresses this issue firstly by including predefined error resilient tools in his core encoding system (part 1) and going straightforward by defining in its $11^{\text {th }}$ part called wireless JPEG 2000 ( JPWL) a set of error resilient techniques to improve the transmission of JPEG 2000 codestreams over error-prone wireless channel.

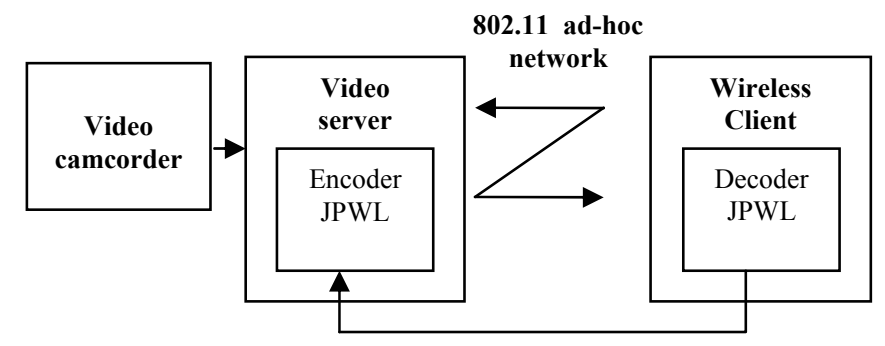

Fig. 1. Wireless video streaming system

\section{JPEG 2000}

JPEG 2000 is the newest image compression standard completing the existing JPEG standard (Taubman \& Marcellin, 2001).

The interest for JPEG 2000 is growing since the Digital Cinema Initiatives (DCI) has selected JPEG 2000 for future distribution of motion pictures. 
Its main characteristics are: lossy or lossless compression modes; resolution, quality and spatial scalability; transmission and progressive image reconstruction; error resilience for low bit rate mobile applications; Region Of Interest (ROI) functionality, etc.

Part 1 of the standard defines different tools allowing the decoder to detect errors in the transmitted codestream, to select the erroneous part of the code and to synchronise the decoder in order to avoid decoder crash. Even if those tools give a certain level of protection from transmission errors, they become ineffective when the transmission channel experiment high bit error rate. Wireless JPEG 2000 (JPEG $200011^{\text {th }}$ part) addressed this issue by defining techniques to make JPEG 2000 codestream more resilient to transmissions errors in wireless systems.

\section{Wireless JPEG 2000 (JPWL)}

Wireless JPEG (JPWL) specifies error resilience tools such as Forward Error correction (FEC), interleaving, unequal error protection.

In this chapter we present a wireless JPEG 2000 video streaming system based on the recommendations of JPWL final draft (JPWL, 2005).

In (Dufaux \& Nicholson, 2004), the description of the JPWL system is presented and the performance of its Error Protection Block (EPB) is evaluated. A fully JPEG 2000 Part 1 compliant backward compatible error protection scheme is proposed in (Nicholson et al, 2003). A memoryless Binary Symmetric Channel (BSC) is used for simulations both in (Nicholson et al, 2003) and (Dufaux \& Nicholson, 2004). However, as packets errors mainly occur in bursts, the channel model considered in those works is not realistic. Moreover JPEG 2000 codestreams interleaving is not considered in (Nicholson et al, 2003).

In this chapter we address the problem of robust and efficient JPEG 2000 images and video transmission over wireless networks. The chapter is organized as follows: In section 2, we present a state of art of wireless JPEG 2000 multimedia communication systems along with the challenges to overcome in terms of codestreams protection against transmission errors. In section 3, we provide an overview of channel coding techniques for efficient JPEG 2000 based multimedia networking. Finally section 4, provides discussions and prospective issues for future distribution of motion JPEG 2000 images and video over wireless networks.

\section{Wireless JPEG 2000 multimedia communication system and its challenges}

In high error rate environments such as wireless channels, data protection is mandatory for efficient transmission of images and video. In this context, Wireless JPEG 2000 (JPWL) the $11^{\text {th }}$ part of JPEG 2000 (JPWL, 2005) different techniques such as data interleaving, Forward Error Correction (FEC) with Reed-Solomon (RS) codes etc. in order to enhance the protection of JPEG 2000 codestreams against transmission errors.

In wireless multimedia system such as the one considered in this chapter (see Figure 1), a straightforward FEC methodology is applying FEC uniformly over the entire stream (Equal Error Correction - EEP). However, for hierarchical codes such as JPEG 2000, Unequal Error Protection (UEP) which assigns different FEC to different portion of codestream has been considered as a suitable protection scheme.

Since wireless channels' characteristics depend on the transmission environment, the packet loss rate in the system also changes dynamically. Thus a priori FEC rate allocation schemes such as the one proposed in (Agueh et al, 2007, a) are less efficient. Two families of data protection schemes address this issue by taking the wireless channel characteristics into 
account in order to dynamically assign the FEC rate for JPEG 2000 based images/video. The first family is based on a dynamic layer-oriented unequal error protection methodology whereas the second relies on a dynamic packet-oriented unequal error protection methodology. Hence, in the first case, powerful RS codes are assigned to most important layers and less robust codes are used for the protection of less important layers. It is worth noting that in this case, all the JPEG 2000 packets belonging to the same layer are protected with the same selected RS code. Examples of layer-oriented FEC rate allocation schemes are available in (Guo et al, 2006) and (Agueh et al, 2007, b). On the other side, in packet-oriented FEC rate allocation schemes such as the one presented in (Agueh et al, 2008), RS codes are assigned by decreasing order of packets importance. In (Agueh et al, 2008), we demonstrate that the proposed optimal packet-oriented FEC rate allocation is more efficient than the layer-oriented FEC rate allocation scheme presented in (Guo et al, 2006) and (Agueh et al, 2007, b). However, layer-based FEC rate allocation schemes have low complexity while packet-oriented FEC allocation methodologies are complex especially when the number of packets in the codestream is high. In this case, packet oriented FEC schemes are unpractical for highly time-constrained images/video streaming applications. In this case switching to a layer oriented FEC rate allocation scheme is more interesting. The smart FEC rate allocation scheme proposed in (Agueh et al, 2009, a) address this issue by allowing switching from a packet oriented FEC scheme to a layer oriented scheme such as the ones proposed in (Agueh et al, 2009, b).

In section 2.1 we present the packet oriented system proposed in (Agueh et al, 2008) to address the issue of robust JPEG 2000 images and video transmission over wireless network. Then in section 2.2 the layer-oriented scheme proposed in (Agueh et al, 2009, b) is described. Finally, in section 2.3 we present the system proposed in (Agueh et al, 2009, a) to unify packet and layer based scheme.

\subsection{Optimal Packet-oriented FEC rate allocation scheme for robust Wireless JPEG 2000 based multimedia transmission}

The functionalities of the proposed JPWL packet-oriented system are presented in Figure 2 The aim of this system is to efficiently transmit a Motion JPEG 2000 (MJ2) video sequence through MANET channel traces.

The system is described as follows:

The input of the JPWL codec is a Motion JPEG 2000 (MJ2) file. The JPEG 2000 codestreams included in the MJ2 file are extracted and indexed.

These indexed codestreams are transmitted to the JPWL encoder (JPWL, 2005) presents a more accurate description of the used JPWL encoder) which applies FEC at the specified rate and adds the JPWL markers in order to make the codestream compliant to Wireless JPEG 2000 standard. At this stage, frames are still JPEG 2000 part 1 compliant, which means that any JPEG 2000 decoder is able to decode them.

To increase JPWL frames robustness, an interleaving mechanism is processed before each frame transmission through the error-prone channel. This is a recommended mechanism for transmission over wireless channel where errors occur in burst (contiguous long sequence of errors). Thanks to interleaving the correlation between error sequences is reduced.

The interleaving step is followed by RTP packetization. In this process, JPEG 2000 codestream data and other types of data are integrated into RTP packets as described in (Schulzrinne et al, 2003). 


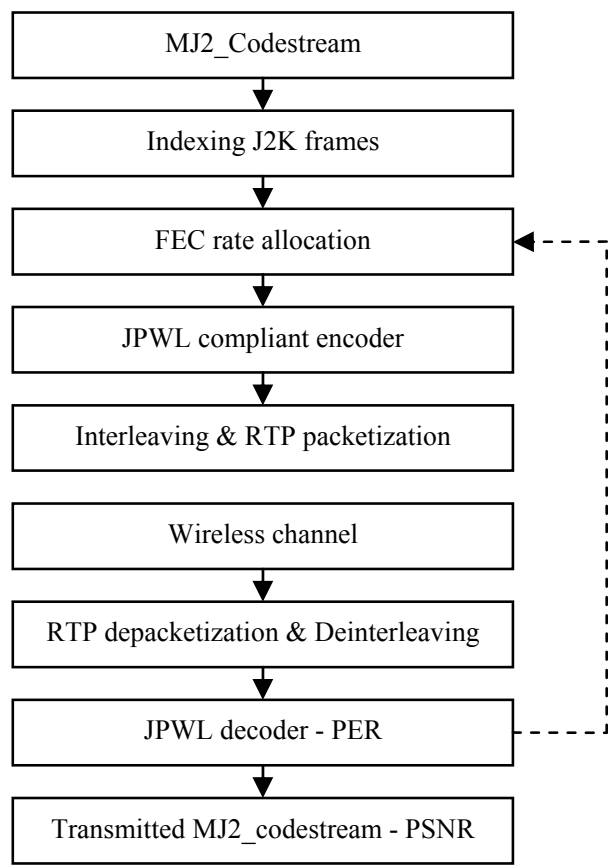

Fig. 2. JPWL based system functionalities

RTP packets are then transmitted through the wireless channel which is modelled in this work by a Gilbert channel model. At the decoder side, after depacketization, the JPWL decoder corrects and decodes the received JPWL codestreams and rebuilds the JPEG 2000 frames. At this stage, parameters such as Packet Error Rate (PER) are extracted, increasing the knowledge of the channel state. The decoder sends extracted parameters back to the JPWL encoder via the Up link. The last process of the transmission chain is the comparison between the transmitted and the decoded image/video. Figure 3 presents JPEG 2000 codestreams transmission through the JPWL packet-oriented FEC system

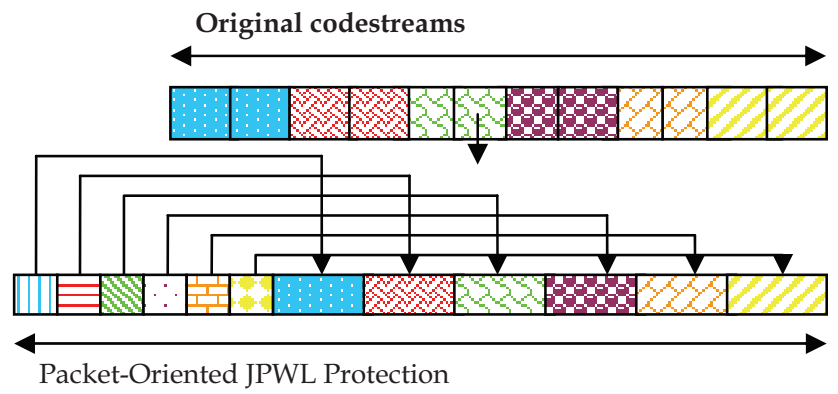

Fig. 3. JPEG 2000 codestreams transmission through the JPWL packet-oriented FEC system 


\subsection{Optimal Layer-oriented FEC rate allocation scheme for robust Wireless JPEG 2000} based multimedia transmission

Unlike the system described in (Agueh et al, 2008), where the FEC rate allocation scheme is packet oriented, in the current system we consider a layer oriented FEC rate allocation scheme. In other words the difference between both systems is the FEC rate allocation module. Actually, in the packet oriented scheme the redundancy is added by taking the packets importance into account (see Figure 3) while in the layer oriented scheme we rely on layers importance to allocate the adequate RS codes (see Figure 4).

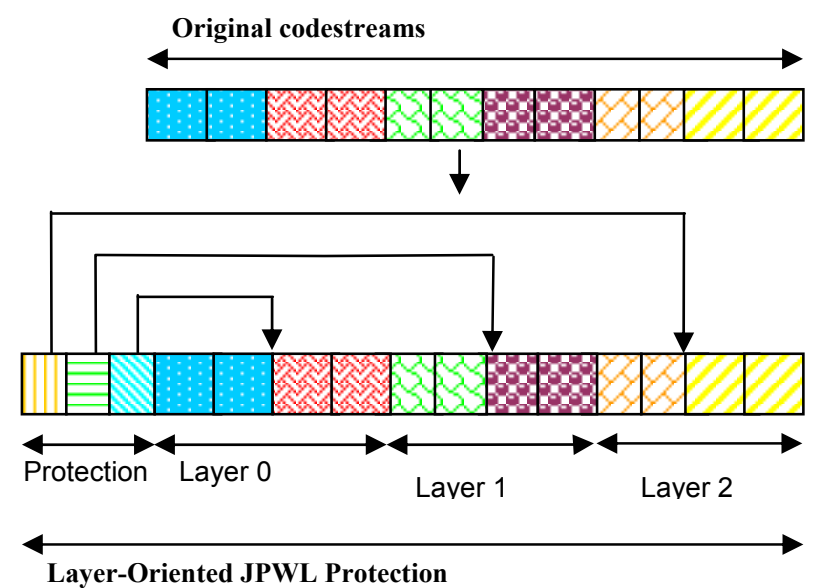

Fig. 4. A JPEG 2000 codestreams transmission through the JPWL layer-oriented FEC system

\subsection{Smart combined Packet/layer based FEC rate allocation scheme for robust Wireless JPEG 2000 based multimedia transmission}

The functionalities of the proposed smart JPWL based system are presented in Figure 5. In this system, indexed JPEG 2000 codestreams are transmitted to the smart FEC rate allocation module. If the number of data packets available in the codestreams is low (typically under the defined smart threshold), the smart module uses the optimal packetoriented FEC rate allocation methodology presented in (Agueh et al, 2008) whereas it switches to the dynamic layer-oriented FEC rate allocation methodology presented in (Agueh et al, 2009, b) when the number of data packets is high. Ones the protection rate determined, the codestreams are transmitted to the JPWL encoder which applies FEC at the specified rate and adds the JPWL markers in order to make the codestream compliant to Wireless JPEG 2000 standard. Hence, Figures 3 and 4 correspond to the JPWL protection where redundant data are added to original codestreams. If the JPEG 2000 Frame which is being processed is constituted by less than a defined threshold (smart_thresh), then the smart FEC rate allocation scheme emulates a scenario similar to the one presented in Figure 3 (packet-oriented FEC rate allocation). Otherwise, it emulates the scenario of Figure 4 (dynamic layer-oriented FEC rate allocation). Protected data are then interleaved and the interleaved codestreams go through the other processes described in section 2.1. 


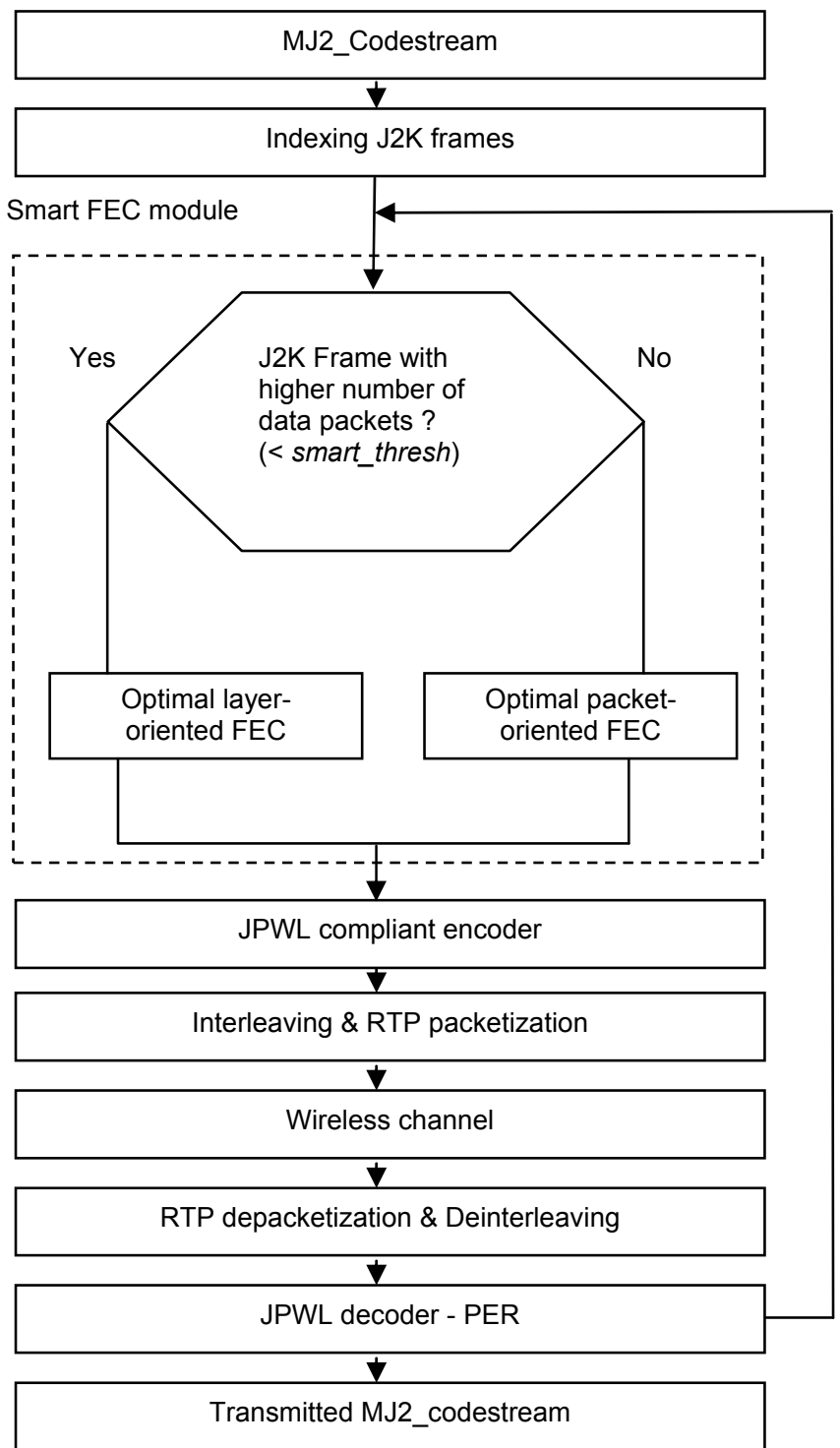

Fig. 5. JPEG 2000 transmission over the smart JPWL system

The interest of the smart FEC rate allocation scheme is to allow switching from the scenario presented in Figure 3 to the scenario described in Figure 4, reducing by this way the complexity of the FEC rate allocation process. Hence, in case of highly layered images/video streaming, the time needed to select the suited FEC rate is significantly reduced. In the following section we formalised the problem of FEC rate allocation, then we present the packet-oriented and layer-oriented algorithm considered in this book. 


\section{Channel coding techniques for robust wireless JPEG 2000 networking}

\subsection{Optimal packet-oriented FEC rate allocation for robust JPEG 2000 image and video transmission over wireless networks}

Making an analogy between the FEC rate allocation problem and the Multiple-Choice Knapsack Problem (MCKP) leads to the conclusion that both problems are NP-hard. Hence, most of the algorithms proposed in the literature such as the one presented in (Thomos et al, 2004), lead to exhaustive search among different FEC rate solutions, exponentially increasing their complexity. These algorithms are thus interesting for an offline video streaming but are unpractical for real-time applications.

To overcome this limitation, Z. Guo et al proposed in (Guo et al, 2006) a slightly complex layered unequal error protection scheme for robust Motion JPEG 2000 streaming over wireless network. However, this algorithm is not JPWL compliant and was designed based on the assumption that the channel is a memoryless Binary Symmetric Channel (uncorrelated error occurrence) which is not realistic because wireless channels have correlated errors sequence. Hence, we have proposed in (Agueh et al, 2007,b) a dynamic layered based unequal error protection FEC rate allocation methodology for efficient JPEG 2000 streaming over MANET. The proposed scheme improved the performance by about $10 \%$ compared to a priori selection of channel coding (Agueh et al, 2007,a). However the main drawback of both methodologies is that the FEC rate allocation is suboptimal. In fact, in both schemes the protection strategy is layer based which implies that a selected FEC rate is applied to all the substreams belonging to the same layer. This limits the effectiveness of those protection strategies especially for fast varying channels where the selected FEC rate may need to be updated from one substream to another.

In (Agueh et al, 2008) we propose a slightly complex, packet based optimal FEC rate allocation algorithm for robust Motion JPEG 2000 video streaming over wireless channel.

\section{1.a Problem formalization}

The goal is to optimally protect JPEG 2000 images/video for robust streaming over wireless channel.

Considering that JPEG 2000 codestreams are constituted by a set of $S$ substreams, the optimal FEC allocation problem can be resumed by answering the question: How to optimally protect each substream so as to minimize the transmitted image distortion under a rate constraint determined by the available bandwidth in the system?

Since the JPEG 2000 standard specifies that packets are byte aligned, it is especially interesting to work with Galois Field $G F\left(2^{8}\right)$ to provide error correction capabilities. In this context, JPWL final draft (JPWL, 2005) recommends the use of Reed-Solomon (RS) codes as FEC codes and fixes a set of RS default codes for substream protection before transmission over wireless channels.

Let $\gamma$ be a substream protection level selected in the range $0 \leq \gamma \leq \gamma_{\max }$, each protection level corresponds to a specific RS code selected between JPWL default RS codes ( $\gamma=0$ means that the substream is not transmitted, $\gamma=1$ means transmission with protection level 1, higher values imply increasing channel code capacity with $\gamma$ ).

Let $B_{a v}$ the byte budget constraint corresponding to the available bandwidth in the system. Let $l_{i}$ the length in bytes of the $i^{\text {th }}$ packet of the $S$ substreams and $R S(n, k)$ the ReedSolomon code used for its protection, the corresponding protection level is $\gamma$ and the FEC 
coding rate is $R=\frac{k}{n}$.We define $f e c=\frac{1}{R_{i}}=\frac{n}{k} \quad$ as the invert of the channel coding rate, so $l_{i}{ }^{*} f e c$ represents, in byte, the increase of the $i^{\text {th }}$ packet length when protected at level $\gamma$. The correct decoding of packet $i$ at the receiver yields a reduction of the distortion on the transmitted image. Let $R D_{i}^{0}$ be respectively the reduction of distortion associated to decoding of packet $i$ and $R D_{i, \gamma}$ the reduction of distortion achieved when packet $i$ is protected at level $\gamma$. The reduction of distortion metric associated to the correct decoding of the packets of a JPEG 2000 codestream is extracted from a codestream index file. The codestream index file is generated by the OpenJPEG library (http://www.openjpeg.org) and defines the gain in quality and the range of bytes corresponding to each packet. The reduction of distortion metric is presented in (Agueh et al, 2008).

We define the gain as the ratio between the image quality improvement $R D_{i, \gamma}$ and the associated cost in terms of bandwidth consumption $l_{i} \times f e c$.

Thus, the FEC allocation problem becomes: How to optimally select substream $i$ protection level $\gamma$ so as to maximize the associated reduction of distortion $R D_{i, \gamma}$ under a budget constraint $B_{a v}$. This problem is formalised by:

$$
\begin{aligned}
& \text { Maximize } \sum_{i=1}^{S} \frac{R D_{i, \gamma}}{l_{i} \cdot f e c_{i}} \\
& \text { Subject to } \sum_{i=1}^{S} l_{i} \cdot f e c_{i} \leq B_{a v}
\end{aligned}
$$

\section{1.b Optimization}

Since the optimization problem can be solved by finding the optimal protection for each substream of JPEG 2000 codestreams under a budget constraint, we define $G_{i, \gamma}$ as the gain in quality of the transmitted image obtained at the receiver side when packet $i$ is decoded.

Let $R D_{i, 1}$ and $R D_{i, \gamma}$ be the reduction of distortion obtained when packet $i$ is transmitted respectively with protection level 1 and with protection level $\gamma$, we have:

$$
R D_{i, 1}=\left(1-P_{\text {pack }}^{i, 1}\right) \cdot R D_{\text {pack }}^{i} \text { and } R D_{i, \gamma}=\left(1-P_{\text {pack }}^{i, \gamma}\right) \cdot R D_{\text {pack }}^{i}
$$

Where $P_{\text {pack }}^{1, \gamma}$ and $P_{\text {pack }}^{i, \gamma}$ are the decoding error probabilities obtained when packet $i$ is protected respectively to level 1 and to level $\gamma$. The resulting gain is:

$$
G_{i, 1}=\frac{R D_{i, 1}}{l_{i}}=\frac{\left(1-P_{\text {pack }}^{i, 1}\right) \cdot R D_{\text {pack }}^{i}}{l_{i}}
$$

Similarly, any transmission between two consecutive protection levels $(\gamma$ and $\gamma+1)$ yields an improvement in terms of reduction of distortion but has a budget cost equal to $\left(f e c_{\gamma+1}-f e c_{\gamma}\right) \times l_{i}$, hence we have:

$$
G_{i, \gamma}=\frac{R D_{i, \gamma}-R D_{i, \gamma-1}}{\left(f e c_{\gamma}-f e c_{\gamma-1}\right) \cdot l_{i}}
$$




$$
G_{i, \gamma}=\frac{\left(P_{p a c k}^{i, \gamma-1}-P_{p a c k}^{i, \gamma}\right) \cdot R D_{\text {pack }}^{i}}{\left(f e c_{\gamma}-f e c_{\gamma-1}\right) \cdot l_{i}}
$$

Protection levels incremental gains $G_{1,1}$ to $G_{S, \gamma}$ are derived for each packet and stored in $S$ different vectors. After merging and reorganizing those vectors, the optimal protection level is derived from the maximum related gain value selected when meeting the rate constraint (Bandwidth available B_av). A detailed description of the processes is available in (Agueh et al, 2008).

\section{1.c Synopsis of the FEC rate allocation scheme and algorithm}

Synopsis of the optimal FEC rate allocation algorithm:

Algorithm:

For each JPEG 2000 image

- Model the channel with a Gilbert model and for each possible protection level $\gamma$, evaluate the probability of incorrect word decoding $P_{\text {pack }}^{i, \gamma}$

- $\quad$ For $i=1$ to $i=S$ (Number of JPEG 2000 packets)

$$
\text { For } \gamma=1 \text { to } \gamma=\gamma_{\max }
$$

Estimate $R D_{i, \gamma}=\left(1-P_{\text {pack }}^{i, \gamma}\right) \cdot R D_{\text {pack }}^{i}$

$$
G_{i, \gamma}=\frac{R D_{i, \gamma}-R D_{i, \gamma-1}}{\left(f e c_{\gamma}-f e c_{\gamma-1}\right) \cdot l_{i}}
$$

$$
V(i)[\gamma]=G_{i, \gamma}
$$

End For

End For

- Merging $V(i)$ vectors protection levels if necessary to ensure that $V(i)$ vectors are constituted of strictly decreasing gains values

- Ordering $V_{\text {_all }}$ on decreasing order of importance values $\left(V_{-}\right.$all_ordered $)$

- Selecting each gain value, corresponding to a specific protection level, up to meeting the rate constraint

- Optimally protect JPEG 2000 packets with the corresponding Reed-Solomon codes End For

\subsection{Optimal Layer-oriented FEC rate allocation for robust JPEG 2000 image and video transmission over wireless networks \\ 3.2.a Problem formalization}

Considering that JPEG 2000 codestreams are constituted by a set of $L$ layers, the optimal FEC allocation problem can be resumed by answering the question: How to optimally protect each layer in order to minimize the transmitted image distortion under a rate constraint determined by the available bandwidth in the system?

Let $l a y_{i}$ the length in bytes of the $i^{\text {th }}$ layer of the $L$ layers and $R S(n, k)$ the Reed-Solomon code used for its protection, the corresponding protection level is $\gamma$ and the FEC coding rate is $R=\frac{k}{n}$.

We define $f e c=\frac{1}{R}=\frac{n}{k}$ as the inverse of the channel coding rate, so $\left(\right.$ lay $\left.y_{i}\right) \times f e c$ represents, in bytes, the increase of the $i^{\text {th }}$ layer length when protected at a level $\gamma$. Unlike packet oriented 
FEC scheme, where the 16 default RS codes are considered in the FEC rate allocation process, in this work we restrict the considered RS codes to those with $f e c \leq 2$. In other words we only consider the first 10 default codes. This assumption make sense in layer oriented FEC rate allocation scheme because adding redundant data which in ratio is more than twice superior to the original layers may overload the networks and drastically increase the losses instead of reducing it.

Let $\gamma$ be a layer protection level selected in the range $0 \leq \gamma \leq \gamma_{\max }^{\text {lay }}$, each protection level corresponds to a specific RS code selected between the 10 JPWL default RS codes ( $\gamma=0$ means that the layer is not transmitted, $\gamma=1$ means transmission with protection level 1, higher values imply increasing channel code capacity with $\gamma$ and $\gamma_{\max }^{\text {lay }}=10$ ).

Let $\beta_{i}$ be the number of data packet constituting the $i^{\text {th }}$ quality layer of a JPEG 2000 codestream, $R D_{\text {lay }_{i}}^{0}$ and $R D_{\text {lay }}^{\gamma}$ be respectively the reduction of distortion associated to the correct decoding of layer $i$ and the reduction of distortion associated to the correct decoding of layer $i$ protected to level $\gamma$.

We rely on this codestream index file to derive $R D_{\text {lay }_{i}}^{0}$ and we associated the decoding error probability estimation process presented in (Yee et Weldon, 1995) in order to derive $R D_{\text {lay }_{i}}^{\gamma}$.

Hence, the layer oriented FEC rate allocation problem is formalised by:

$$
\begin{gathered}
\text { Maximize } \sum_{i}^{L} \frac{R D_{\text {lay }_{i}, \gamma}}{\left(\operatorname{lay}_{i}\right) \times f e c_{i}} \\
\text { Subject to } \sum_{i}^{L}\left(\operatorname{lay}_{i}\right) \times f e c_{i} \leq B_{a v}
\end{gathered}
$$

\section{2.b Optimization}

We define $G_{\text {lay }}^{\gamma}$ as the gain in quality of the transmitted image obtained at the receiver side when layer $i$ is decoded.

We derive $R D_{\text {lay }}^{1}$ and $R D_{\text {lay }}^{\gamma}$ the reduction of distortion obtained when layer $i$ is transmitted respectively with protection level 1 and with protection level $\gamma$, we have:

$$
\begin{aligned}
& R D_{\text {lay }_{i}}^{1}=\left(1-P_{\text {lay }_{i}}^{i, 1}\right) \times R D_{\text {lay }_{i}}^{0} \\
& R D_{\text {lay }_{i}}^{\gamma}=\left(1-P_{\text {lay }_{i}}^{i, \gamma}\right) \times R D_{\text {lay }_{i}}^{0}
\end{aligned}
$$

Where $P_{\text {lay }_{i}}^{1}$ and $P_{\text {lay }_{i}}^{\gamma}$ are the decoding error probabilities obtained when layer $i$ is protected respectively to level 1 and to level $\gamma$.

The resulting gain is:

$$
G_{\text {lay }_{i}}^{1}=\frac{R D_{\text {lay }_{i}, 1}}{\operatorname{lay}_{i}}=\frac{\left(1-P_{\text {lay }_{i}}^{1}\right) \times R D_{\text {lay }_{i}}^{0}}{\operatorname{lay}_{i}}
$$

Similarly, any transmission between two consecutive protection levels $(\gamma-1$ and $\gamma$ ) yields an improvement in terms of reduction of distortion but has a budget cost equal to $\left(f e c_{\gamma}-f e c_{\gamma-1}\right) \times \operatorname{lay}_{i}$, hence we have: 


$$
\begin{gathered}
G_{\text {lay }_{i}}^{1}==\frac{R D_{\text {lay }_{i}}^{\gamma}-R D_{\text {lay }_{i}}^{\gamma-1}}{\left(f e c_{\gamma}-f e c_{\gamma-1}\right) \text { lay }_{i}} \\
G_{\text {lay }_{i}}^{\gamma}==\frac{\left(P_{\text {lay }_{i}}^{\gamma-1}-P_{\text {lay }_{i}}^{\gamma}\right) R D_{\text {lay }_{i}}^{0}}{\left(f e c_{\gamma}-f e c_{\gamma-1}\right) \times \text { lay }_{i}}
\end{gathered}
$$

Applying the optimization process proposed in (Agueh et al, 2008), we derive the corresponding FEC rate for each layer. Since the Unequal Error protection is applied at layer level, the FEC rate is selected by decreasing order of layer importance. It is worth noting that all the packets belonging to the same layer are protected at the same FEC rate.

\section{2.c Contribution of the optimal layer oriented FEC rate allocation scheme}

Even if the gain metrics presented in the previous section seem close to the ones used in (Agueh et al, 2008), they hold a fundamental difference because they rely on the contribution of each layer to the reduction of distortion instead of just taking into account the contribution of a specific packet. Actually, during the source coding process the incremental contribution from the set of image codeblocks are collected in quality layers. Due to the fact that the rate-distortion compromises derived during JPEG 2000 truncation process are the same for all the codeblocks, for any quality layer index $i$ the contributions of quality layer 1 through quality layer $i$ constitute a rate-distortion optimal representation of the entire image. Hence, at layer level the reduction of distortion values are strictly decreasing. In contrast, the selection of a specific JPEG 2000 packet does not guarantee that the contributions of packet 1 to the selected index packet are monolithically decreasing. In this case, as confirmed by Descampe et al in (Descampe et al, 2006), some additional restrictions have to be added to the considered convex-hull in order to ensure rate-distortion and cost-distortion optimality. This justifies the necessity to have a merging step in the packet oriented FEC scheme (Agueh et al, 2008). Actually, it ensures that the convex-hull is always convex. In the layer oriented FEC this step is skipped because the reduction of distortion curve is already monolithically decreasing, significantly reducing the complexity and thus the time-consumption of the FEC rate allocation algorithm. Moreover, in the optimal layer oriented FEC scheme we only consider the first 10 RS codes instead of considering all the 16 default RS codes defined by JPWL standard as it is the case in (Agueh et al, 2008). This also considerably reduces the FEC scheme time consumption as its leads to less gains values computation which makes the proposed optimal layer FEC rate allocation scheme a good candidate for real time images/video streaming applications.

In addition, the number of layers available in the codestreams is another criterion which contributes to the reduction of the time consumption of our proposed FEC scheme. Actually, a JPEG 2000 image extracted from a Motion JPEG 2000 video sequence is defined by ( $L, R, C)$ where $L$ is the number of quality layers of the considered image, $R$ is its resolution level corresponding to the decomposition levels of the Discrete Wavelet Transform and $C$ is the number of components. Assuming that the considered JPEG 2000 image is not spatially divided and thus is described by a unique tile, the number of data packets available in the considered JPEG 2000 codestreams is given by $S=L \times R \times C$. In this context, the complexity of packet oriented FEC schemes is based on the $S$ data packets while the complexity of the optimal layer based FEC is based on the L layers available in the 
codestreams. In scalable JPEG 2000 images, since the number of layers is significantly lower in comparison to the number of data packets, the time consumption of our proposed layer oriented FEC scheme is significantly low in comparison to packet oriented scheme.

\section{2.d Algorithm}

For each JPEG 2000 image

- Model the channel with a Gilbert model and for each possible protection level $\gamma(0 \leq \gamma \leq 10)$, evaluate the probability of incorrect word decoding $P_{\text {lay }_{i}}^{\gamma}$

- $\quad$ For $i=1$ to $i=L$ (Number of JPEG 2000 layers)

For $\gamma=1$ to $\gamma=10$

$$
\begin{aligned}
G_{\text {lay }_{i}}^{1}== & \frac{R D_{\text {lay }_{i}}^{\gamma}-R D_{\text {lay }_{i}}^{\gamma-1}}{\left(f e c_{\gamma}-f e c_{\gamma-1}\right) \text { lay }_{i}}
\end{aligned}
$$

End For

\section{End For}

- Ordering gain values in decreasing order of importance

- Selecting each gain value, corresponding to a specific protection level, up to meeting the rate constraint

- Optimally protect JPEG 2000 layers with the corresponding RS codes End For

\section{2.e Performance of layer based FEC scheme in terms of time consumption}

In Figure 6 the run time of the proposed layer based FEC rate allocation scheme is plotted versus the number of data packets available in the JPEG 2000 codestreams. This curve is compared to the one achieved using the optimal packet oriented FEC rate allocation scheme (Agueh et al, 2008). These results are achieved using an Intel core Duo CPU $2.9 \mathrm{Ghz}$ Workstation.

As packet-oriented and layer oriented schemes are linked by the number of layers available in each image, we vary this parameter in order to derive some comparable results. In the considered scenario, the number of available resolution and component of JPEG 2000 frames are fixed (resolution $=10$ and component $=1$ ) because these parameters do not impact the time-performance of layer oriented FEC rate allocation schemes. In Figure 6 each packet (i) corresponds to a specific JPEG 2000 frame (with a specific quality layer).

In this scenario, the available bandwidth in the system is set to $18 \mathrm{Mbits} / \mathrm{s}$ $\left(B_{a v}=18 \mathrm{Mbits} / \mathrm{s}\right.$ ). It is worth noting that in practice few existing JPEG 2000 codecs allow high quality scalability and to our knowledge, none of them can handle more than 50 quality layers. Hence, the considered scenario allows generalization to future high quality layer scalable FEC rate allocation systems.

In Figure 6 we notice that both layer and packet oriented scheme have a run time linearly increasing with the number of packets available in the codestreams. However, the optimal layer based FEC scheme is significantly less time consuming than the packet based FEC scheme. For codestreams containing less than 1000 packets (quality layers $\leq 10$ ) the packet oriented FEC scheme is 3 times more time consuming than our optimal layer based FEC scheme. For JPEG 2000 codestreams, whose number of packets is between 1000 and 5000 
(quality layers between 10 and 50) the packet oriented scheme is up to 5 times the run time of the layer based FEC scheme. Since existing JPEG 2000 codecs handle less than 50 quality layers, our proposed optimal layer based scheme is a good candidate for real-time JPEG 2000 codestreams over wireless channel as its yields low time consumption.

The proposed optimal layer based scheme, due to its low time consumption, could be viewed as a good candidate for future high quality layer scalable wireless JPEG 2000 based images and video streaming applications.

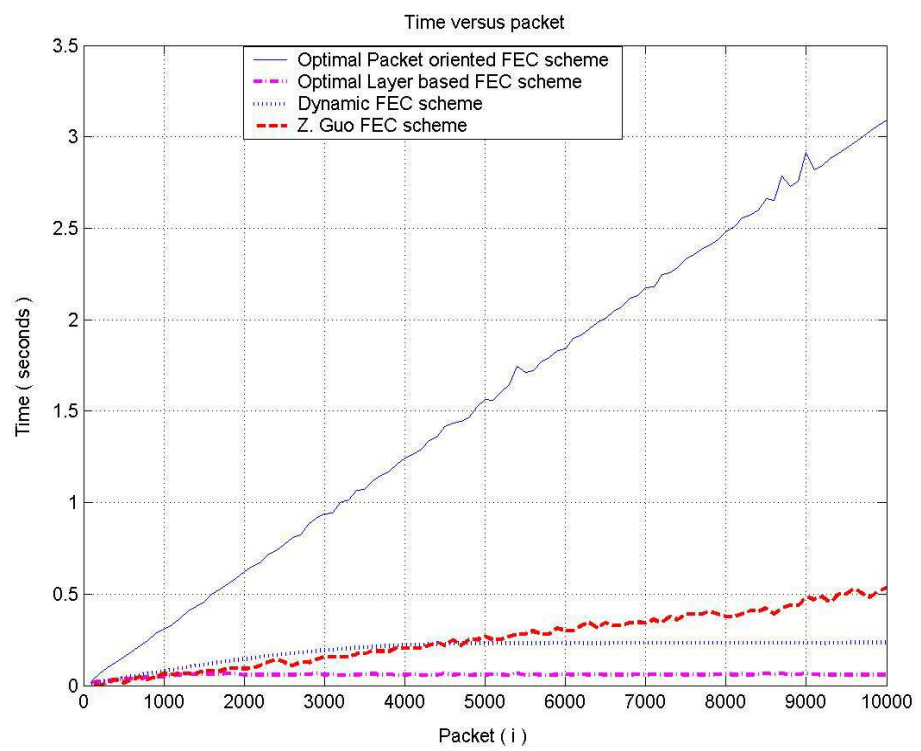

Fig. 6. Time versus packets: Fixed image resolution $(\mathrm{R}=10)$-Varying quality layers (0 to 100) - One component $(\mathrm{C}=1)$

Although the layer based scheme achieves good performances in terms of time consumption in comparison to packet oriented FEC rate allocation schemes, the last ones present better performance in terms of visualization quality especially for highly noisy channels. In the following section we demonstrate the effectiveness of the optimal layer based FEC scheme thanks to a client/server application of Motion JPEG 2000 video streaming over real ad-hoc network traces.

\section{2.f Packet-oriented and Layer-oriented FEC rate allocation for Motion JPEG 2000 video streaming over real ad-hoc network traces}

In this section we present the results achieved while streaming Motion JPEG 2000 based video over real ad-hoc network channel traces (Loss patterns acquired during the WCAM Annecy 2004 measurement campaigns IST-2003-507204 WCAM, Wireless Cameras and Audio-Visual Seamless Networking, 2004) and we demonstrate that the proposed optimal layer based scheme outperforms existing layer oriented FEC schemes even if for highly noisy channel it is less efficient than packet oriented FEC scheme. The comparison is handled both in terms of Structural Similarity (SSIM) (Wang et al, 2004) and in terms of successful decoding rate. We derive the Mean SSIM metric of the Motion JPEG 2000 video 
sequence by averaging the SSIM metrics of the JPEG 2000 images contained on the considered video sequence. It is worth noting that each SSIM measure derived is associated to a successful decoding rate metric which corresponds to decoder crash avoidance on the basis of 1000 transmission trials.

The considered wireless channel traces are available in (Loss Patterns, 2004) and the video sequence used is speedway.mj2 (Speedway, 2005) containing 200 JPEG 2000 frames generated with an overall compression ratio of 20 for the base layer, 10 for the second layer and 5 for the third layer. Figure 7 presents the successful decoding rate of the motion JPEG 2000 video sequence speedway.mj2 (Speedway, 2005) transmission over real ad-hoc network channel traces (Loss Patterns, 2004). We observe that for highly noisy channels ( $C / N \leq 15 d B$ ), the proposed optimal layer outperforms other layer based FEC schemes but is less efficient than the packet oriented scheme. For noisy channel $(15 d B \leq C / N<18 d B)$, we notice that all layer based UEP schemes exhibit similar performances in terms of successful decoding rate. For low noisy channel $(C / N \geq 18 d B)$ all the FEC schemes yield the same improvement in terms of successful decoding rate.

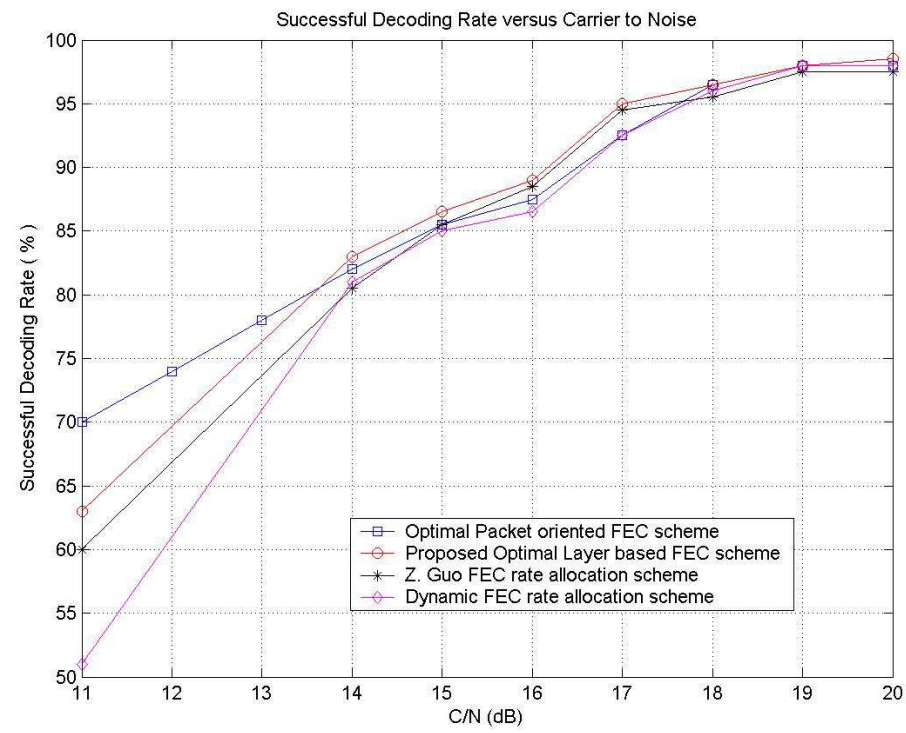

Fig. 7. Successful decoding rate versus Carrier to Noise Ratio

In Figure 8 we show that our proposed optimal layer based FEC rate allocation scheme still outperforms other layer based schemes in terms of Mean SSIM. This is due to the fact that the base layer which is the most important part of the codestreams is highly protected in our proposed scheme, in comparison to other layer based schemes, guaranteeing this way a good quality for the visualization.

It is worth noting that, for highly noisy channels, our optimal layer oriented FEC scheme is less efficient than optimal packet oriented FEC scheme presented in (Agueh et al, 2008). However the last one is unpractical for real time streaming applications when the number of packets in the codestreams is high. In contrast our proposed layer oriented efficiently overcomes this limitation. In this context, instead of being used to replace packet oriented FEC rate allocation schemes, our proposed optimal layer based FEC scheme should be used to complete it. 


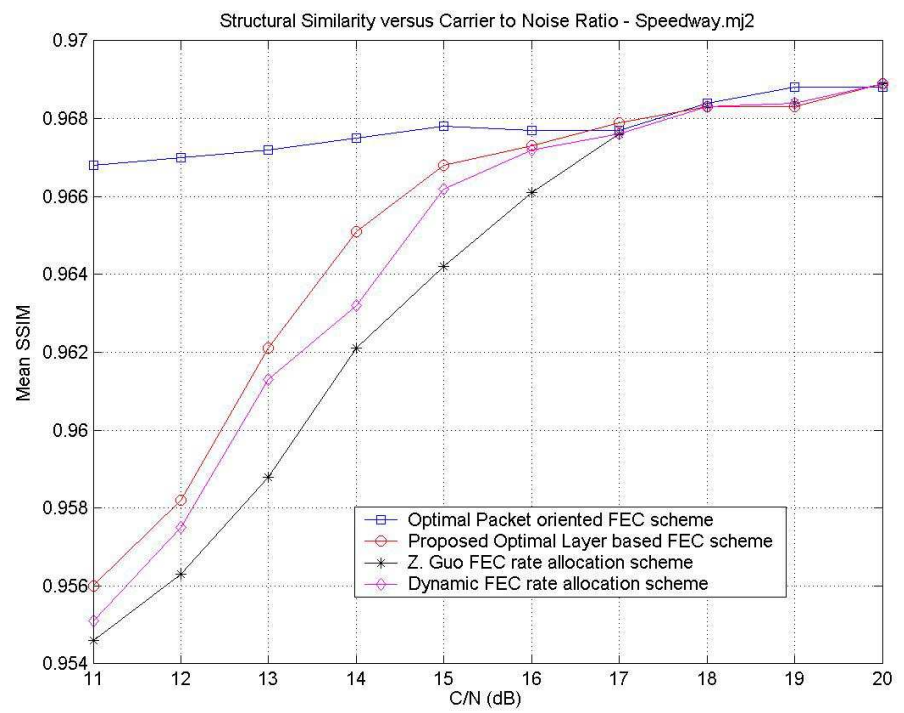

Fig. 8. Mean Structural Similarity versus Carrier to Noise Ratio

\section{Discussion and prospective issues}

\subsection{On JPEG 2000 codestreams interleaving}

In this section, we discuss and evaluate the impact of data interleaving in the effectiveness of the FEC rate allocation scheme. Thanks to the interleaving matrix presented in Figure 9, protected JPEG 2000 data are decorrelated before being sent through the wireless channel. Hence, the impact of consecutive channel errors sequences on the transmitted codestreams is reduced. In Figure 9 the protected JPEG 2000 codestream is divided into $P x$ packets of length $N$. Then, the interleaving process consists in storing $M$ consecutive packets into a $M \times N$ matrix and to read the columns of this matrix so that two initially consecutive symbols are separated by a distance of $I=M$ (symbols). We refer to $I$ as the interleaving degree. The considered channel is a real mobile ad-hoc network channel experiencing $P E R=3.88 \times 10^{-2}$ and the interleaving degrees are $1,2,4,8,16,32,64$ and 128 . Table 1 shows the PSNR evolution as function of interleaving degree $I$. The considered image is speedway_0.j2k protected with the optimal packet-oriented JPWL compliant scheme.

The interest of interleaving is shown in table 1 in the sense that the PSNR and the successful decoding rate increase with the interleaving degree $I$. The results in table 1 are valid for a Gilbert channel with a specific error correlation factor and are no longer the same when this factor changes. For the considered channel, we observe that for $I \leq 8$, interleaving has no noticeable impact because the interleaving degree $I$ is smaller than the average error burst length. In fact, we show in (Agueh et al, 2008) that the upper bound of the mean error burst length is $L_{B}^{\max }=10$ bytes. Hence, in order to be efficient, the interleaving degree should be higher than 10 bytes. When $I$ is increased to 16 or more, we notice an improvement of both the PSNR and the successful decoding rate. However, we observe that higher values of $I$ 
(128) yield only slight improvement in terms of PSNR while consuming considerable memory resources leading to the conclusion that reasonable interleaving degree (typically $I=16$ or $I=32)$ is a good compromise.

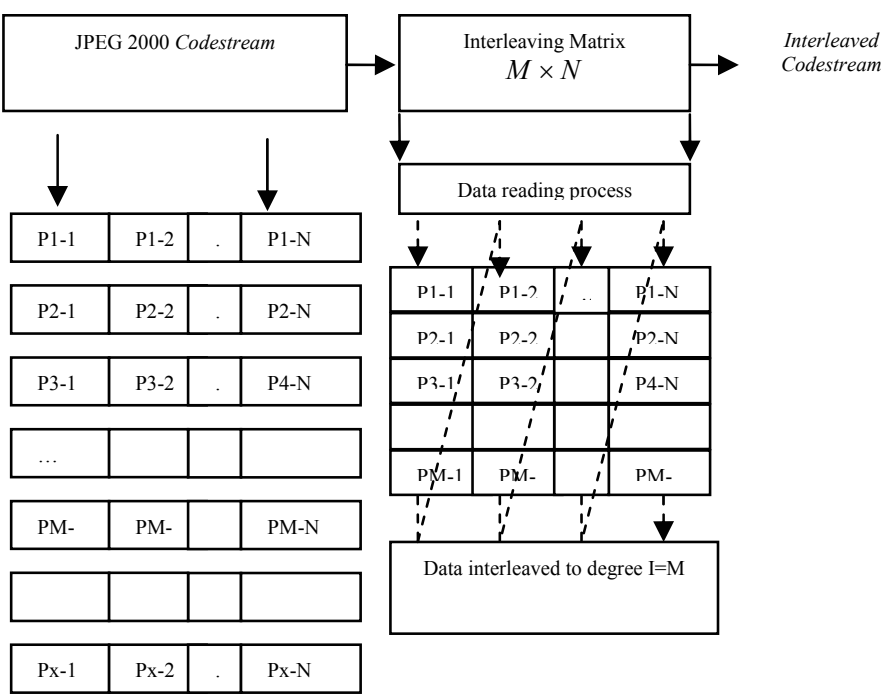

Fig. 9. Interleaving process

\begin{tabular}{|c|c|c|}
\hline $\begin{array}{c}\text { Interleaving degree } \\
\mathrm{I}\end{array}$ & PSNR $(\mathrm{dB})$ & $\begin{array}{c}\text { Successful } \\
\text { Decoding Rate }\end{array}$ \\
\hline $\mathrm{I}=1$ & 24.1 & 77.5 \\
\hline $\mathrm{I}=2$ & 24.6 & 89.8 \\
\hline $\mathrm{I}=4$ & 25.2 & 92.1 \\
\hline $\mathrm{I}=8$ & 31.8 & 93.4 \\
\hline $\mathrm{I}=16$ & 38.7 & 94.5 \\
\hline $\mathrm{I}=32$ & 44.33 & 94.7 \\
\hline $\mathrm{I}=64$ & 44.38 & 94.9 \\
\hline $\mathrm{I}=128$ & 44.37 & 94.8 \\
\hline
\end{tabular}

Table 1. Interleaving degree and associated image PSNR

Even if the empirical selection of interleaving degree is commonly accepted, it is worth noting that optimal dynamic selection of interleaving degree is still an interesting and open issue. Moreover, proposing new interleaving schemes which are able to take into account the specificity of JPEG 2000 codestreams is also an open issue. Fabrizio \& Baruffa address this issue in (Fabrizio \& Baruffa, 2005) by proposing a backward-compatible interleaving technique for robust JPEG 2000 wireless transmission. To the best of our knowledge, the virtual interleaving scheme proposed by Fabrizio \& Baruffa is the only JPEG 2000 based backward compatible interleaving scheme available in the literature. Hence, original and new interleaving scheme are still needed to improve the robustness of JPEG 2000 codestreams against transmission errors in wireless networks. 


\subsection{Scalable JPEG 2000 transmission}

Many problems are still to be addressed in the framework of JPEG 2000 codestreams transmission over wireless networks. Image scalability based on dynamic available bandwidth estimation is one of those problems. In the literature, proposed image scalable systems have been implemented using a fixed available bandwidth in their considered scenarios (Li and Chang, 2009), (Devaux et al, 2007). This assumption is no longer true in wireless systems because they rely on radio waves whose characteristics depend on the transmission environment. Moreover, few of the proposed systems addressed simultaneously the bandwidth estimation problem and the issue of smoothness for JPEG 2000 codestreams scalability. In (Mairal \& Agueh, 2010), we addresses both issues by proposing a scalable and non aggressive wireless JPEG 2000 image and video transmission algorithm based on a dynamic bandwidth estimation tool.

The main limitation of the scalable system proposed in (Mairal \& Agueh, 2010) is that it handles only one wireless client (see section 4.2.a). However, this limitation could be overcome by generalizing the proposed algorithm to multiple wireless clients' scenario. We propose in section 4.2.b, a framework for this generalization which opens the way for efficient wireless JPEG 2000 codestreams transmission in Next Generation Networks which are characterize by the cohabitation of multiples wireless devices having different standards requirements and different capacities.

\section{2.a Scalable JPEG 2000 transmission for single wireless client}

In the this section we present an adaptive bandwidth estimation tool and propose an additional scalability tool at the encoder, which dynamically and efficiently selects the best resolution and layer for each JPEG 2000 frame before transmission through the wireless channel. Hence, according to the estimated bandwidth, refinement layers could be added or removed from JPEG 2000 codestreams. When required, scale changes are gradual and smooth in order to guaranty a comfort in terms of visualization. We present in the following the processes, which are implemented at the encoder.

Algorithm:

Once connected, the server starts the WBest process in order to obtain the initial available bandwidth. WBest is the available bandwidth estimation tool implemented in the system. A detailed description of WBest is provided in ( $\mathrm{Li}$ and Chang, 2009). At this step the goal is to send images and video with maximum detail (highest resolution and all refinement layers) matching with the estimated bandwidth. The original resolution and number of layers of the considered video is found using an indexer like the one available in OpenJpeg (www.openjpeg.org). In (Mairal \& Agueh, 2010), the default number of resolutions is 6, the length and the width of the image must be a power of 2 (here $352 \times 288$ ), the number of layers is 3. Let $l$ be a layer of a JPEG 2000 image and $S E_{\text {rate }}^{l}$ is corresponding source encoding rate. Let $f e c_{\text {rate }}^{l}=\frac{n}{k}$ be the inverse of the Reed-Solomon code RS(n,k) selected by the FEC rate allocation scheme to protect layer lagainst transmission errors. Let frame_length be the amount of data needed to transmit layer $l$ protected. We have:

$$
\text { frame_length }=H \times W \times S E_{\text {rate }}^{l} \times f e c_{\text {rate }}^{l}
$$

The proposed scheme is able to adapt to channel conditions thanks to the bandwidth estimation tool. Hence, when the channel experienced good conditions, our heuristic selects 
the highest resolution with the highest quality (all the refinement layers are transmitted). If the channel experienced harsh conditions, image layers and resolution are decreased up to defined thresholds. We empirically set thresholds $\left(l_{\max } / 2\right)$ and $\left(\right.$ resol $\left._{\min }^{\text {desired }}\right)$ as respectively the minimum layer downscaling allowed and the minimal resolution, which guaranties comfort in terms of image visualization. Contrarily, $l_{\min }$ and $r e s o l_{\min }$, respectively the base layer and the minimal resolution possible do not guaranty a visual comfort. Hence, when the channel experienced bad conditions, image layers are incrementally reduced while maintaining original resolution of the JPEG 2000 frame to highest level. However, is the corresponding frame length do not match the available bandwidth, image resolution downscaling is processed. It is worth noting that our fixed thresholds are valid for our scenarios and may change in different environments.

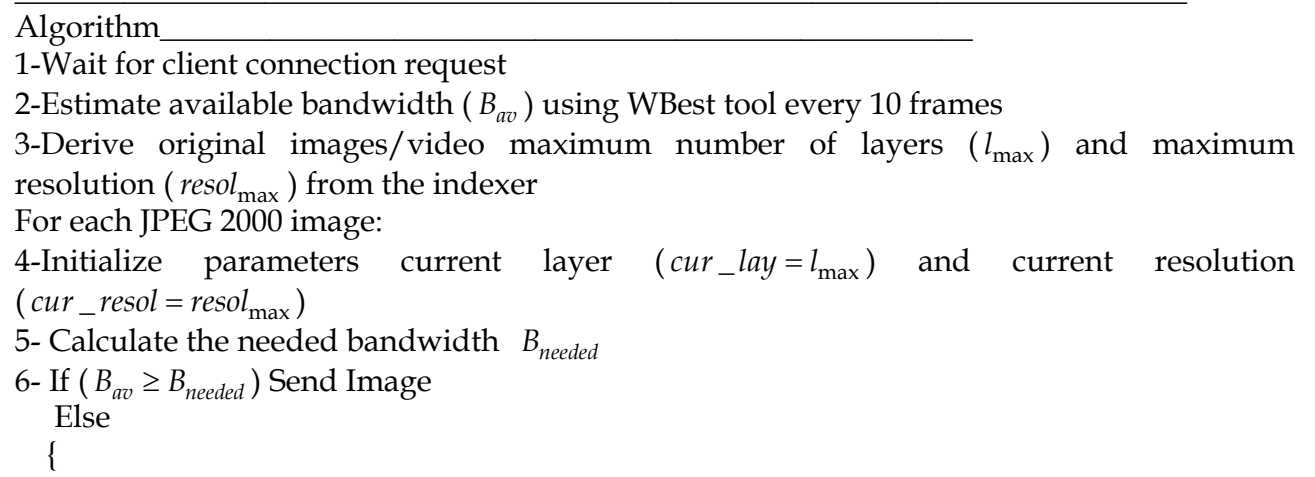

Step 1: Guaranty of comfort during visualization

While (cur_lay $>l_{\max } / 2$ and cur_resol $>$ resol min $_{\text {desired }}$ )

$\left\{\right.$ Estimate $B_{\text {needed }}=\sum_{i=1}^{\text {cur_lay }}$ cur_resol $\times S E_{\text {rate }}^{i} \times f e c_{\text {rate }}^{i}$

Increase layer to remove ( lay_to_rem $=$ lay_to_rem +1$)$ and

Fixe resolution or fixe layer to remove and decrease resolution

$($ cur_resol $=$ cur_resol -1$)$ until reaching $\left(B_{a v} \geq B_{\text {needed }}\right)$

\}

Step 2: Without guaranty of comfort during visualization

While (cur_lay $>0$ and cur_resol $>$ resol $\left.l_{\min }\right)$

$\left\{\right.$ Estimate $B_{\text {needed }}=\sum_{i=1}^{\text {cur_lay }}$ cur_resol $\times S E_{\text {rate }}^{i} \times f e c_{\text {rate }}^{i}$

Increase layer to remove ( lay_to_rem $=$ lay_to_rem +1 ) and

Fixe resolution or fixe layer to remove and decrease resolution

(cur_resol $=$ cur_resol -1$)$ until reaching $\left(B_{a v} \geq B_{\text {needed }}\right)$

\}

\}

7- Smooth scale changes in order to avoid sudden and temporal image variation (average among 5 previous frames parameters) 
Once the resolution and the number of layers are chosen, the server sends the video streaming to the client.

The available bandwidth estimation tool is launched every 10 frames but this frequency could be changed according to the application requirements.

In our work, we evaluate the extra time yielded by different refreshment frequencies while transmitting speedway.mj2 video. We have:

$$
\Delta t(r e f r)=75 e^{-0.115 r e f r}(\text { seconds) }
$$

Where $\Delta t$ and refr are respectively the extra time and the number of transmitted frames between two consecutive bandwidth estimations. We derive from this study that a refreshment of 10 frames increases less than $20 \%$ the total transmission time while guaranteeing sufficiently accurate channel tracking.

An interesting extension to this work could be to optimally adapt the frequency of the bandwidth estimation tool to the channel conditions.

The efficiency of the proposed heuristic is demonstrated using a wireless client/server video streaming application. In the following section, we present the results derived from different video streaming scenarios.

The video streaming scenarios considered in this work are derived from wireless transmission trials used in the literature for bandwidth estimation purpose. WBest is the available bandwidth estimation tool implemented in our system.

The video sequence is speedway.mj2, which is a 352×288 motion JPEG2000 sequence constituted of 200 JPEG 2000 frames with six resolutions and three layers each.

\section{Scenario}

In the considered scenario, the wireless channel considered is derived from BART tool (Johnsson \& Björkman, 2008), which estimates the available bandwidth in an end-to-end path where the bottleneck is a wireless hop. It uses the Probing Packet Pair Trains Dispersion Technique and improves the system using Kalman filters to measure and track the changes.

In this scenario, we focus on the fast varying part of the estimated bandwidth. Moreover, we divide the bandwidth estimated by BART tool 0 . by a parameter $\delta=2$ in order to show that our scheme is efficient even when the wireless channel experienced harsh conditions.

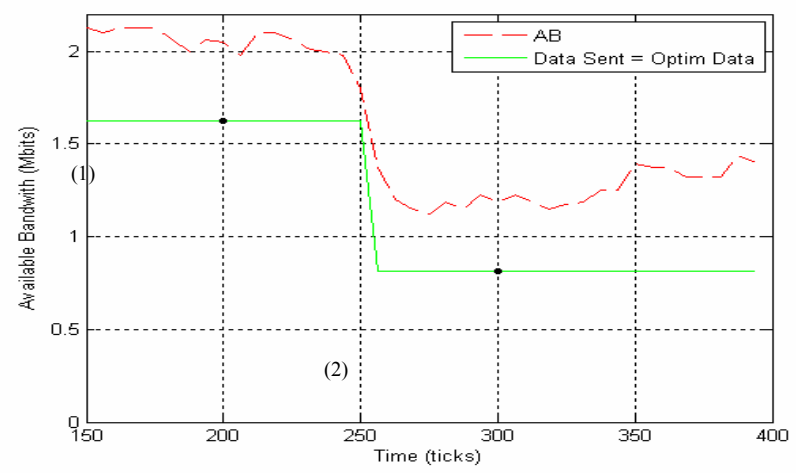

Fig. 10. Available bandwidth versus time - BART Tool scenario 


\begin{tabular}{|c|c|c|c|}
\hline Scenario & $\begin{array}{c}\text { Image } \\
\text { Length }\end{array}$ & $\begin{array}{c}\text { Image } \\
\text { Width }\end{array}$ & $\begin{array}{c}\text { Layer of transmitted } \\
\text { Images }\end{array}$ \\
\hline$(1)$ & 352 & 288 & 3 \\
\hline$(2)$ & 352 & 288 & 2 \\
\hline
\end{tabular}

Table 2. Scalability parameters - BART Tool scenario

In Figure 10, point (1) indicates that the estimated bandwidth is higher than the needed bandwidth, hence initial JPEG 2000 frames are transmitted. Point (2) shows that the estimated bandwidth is decreased and becomes insufficient to send original images. Hence, the algorithm maintains the resolution at the highest level (initial value) but one layer is removed from original frames as shown in Table 2.

In this section, the performance of scalable JPEG 2000 transmission over wireless networks is outlined. In the following section we address the problem of JPEG 2000 codestreams scalability in a context of multiple wireless clients networking.

\section{2.b Scalable JPEG 2000 transmission for multiple wireless clients}

In order to address the issue of simultaneous service provisioning for multiple wireless receivers, a multithread server is implemented at the encoder.

After detecting the value of Max_Client the number of wireless receivers in a considered cell, the server waits for client's connection requests. Once connected and identified, a receiver sends the maximal resolution of its viewer to the server so that the adequate size and image quality layers could be selected. Then, the available bandwidth estimation tool is launched to obtain an initial estimation of the downlink capacity. After the available bandwidth estimation, the algorithm presented in (Mairal \& Agueh, 2010) selects the suitable size and number of layers of the images to be sent.

In the following we present the processes, which are implemented at the encoder.

\section{Algorithm:}

1. While (number of connected clients (connected_clients) $\leq$ Max_Client )

\{

2. Server waits for a client to connect to his socket

3. Server starts a new thread to serve the new wireless client

Increment the number of connected clients (connected_clients); \}

4. Receive wireless client equipment resolution (max_device_resolution)

5. While (transmitting JPEG 2000 images/video)

\{

Every 10 frames

\{

Launch WBest and estimate available Bandwidth $\left(B_{a v}\right)$

Select the suitable scalability parameters

\}

Send frames with selected parameters

Increment the number of transmitted frames (Sent_frames); 
The selected size is never bigger than the wireless device's resolution. The clients connected to the server receive the highest images/video quality achievable for the estimated available bandwidth. The available bandwidth estimation tool is launched every 10 frames but this frequency could be changed according to the application requirements. As recommended in (Mairal \& Agueh, 2010), we fix this frequency to 10 frames. The result is a smooth and robust video sequence for each receiver. It is worth noting that the original JPEG 2000 codestreams is copied and stored at the encoder so that new JPEG 2000 codestreams are generated for each client according to the parameters selected by the proposed scalability algorithm. An interesting extension to this work is to implement a real time system which is able to handle real time multiple JPEG 2000 source coding.

\section{Scenario}

The video streaming scenarios considered in this work are derived from wireless transmission trials used in the literature for bandwidth estimation purpose. WBest is the available bandwidth estimation tool implemented in our system.

The video sequence is speedway.mj2 (Speedway, 2005) which is a $352 \times 288$ motion JPEG2000 sequence constituted of 200 JPEG 2000 frames with six resolutions and three layers each.

In the considered scenario (figure 11), an IEEE 802.11 based wireless network with two receivers and one sender connected to an access point is used to demonstrate the effectiveness of the proposed system.
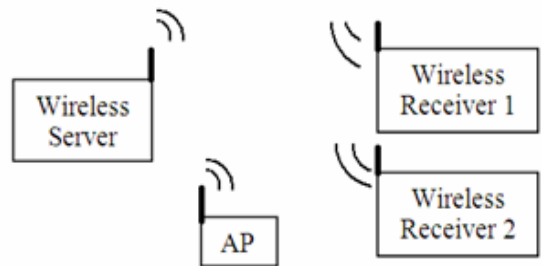

Fig. 11. Single server / multiple wireless clients' transmission scenario

In Table 3, we present the results of the considered scenario where speedway.mj2 is transmitted to multiple wireless clients. The estimated available bandwidths along with the characteristics of the selected stream for each client are presented. In the first case, the client receives a video composed of JPEG 2000 frames with all layers and which size is 176x144.

In this scenario, the size of transmitted video sequence is decreased in comparison to the original JPEG 2000 codestreams because the estimated available bandwidth is not higher enough to allow full original video transmission. The proposed algorithm helps preserving the details by guarantying a comfort of visualization. As we recommend in (Mairal \& Agueh, 2010), when more than half of the original layers have to be removed to fit bandwidth requirements, images quality layers is maintained while their size is decreased. Hence, for the first receiver, instead of transmitting JPEG 2000 images with 352x176 sizes with only the base layer, the server transmits images with all the layers after decreasing the resolution. It is worth noting that in the considered scenario, maintaining the same size as user equipment settings is not mandatory.

For the second receiver (see Table 3), the video transmitted is a sequence of JPEG 2000 frames with the all the layers, but with a decreased resolution of $88 \times 72$. 
The third column of Table 3, indicates the channel occupation during the transmission. We can observe that the available bandwidth is never exceeded.

\begin{tabular}{|c|c|c|c|c|}
\hline Frames & Parameters & Receiver 1 & Receiver 2 & Total \\
\hline \multirow{4}{*}{0 to 40} & $\begin{array}{l}\text { Estimated } \\
\text { Available BW }\end{array}$ & 744 kbits & 704 kbits & 1448 kbits \\
\hline & $\begin{array}{l}\text { Amount of data } \\
\text { sent }\end{array}$ & 405504 bits & 101376 bits & 506880 bits \\
\hline & $\begin{array}{l}\text { Number of } \\
\text { layers }\end{array}$ & 3 & 3 & -- \\
\hline & Resolution & $176 \times 144$ & $88 \times 72$ & -- \\
\hline \multirow{4}{*}{40 to 199} & $\begin{array}{l}\text { Estimated } \\
\text { Available BW }\end{array}$ & 704 kbits & 704 kbits & 1408 kbits \\
\hline & $\begin{array}{l}\text { Amount of data } \\
\text { sent }\end{array}$ & 405504 bits & 101376 bits & 506880 bits \\
\hline & $\begin{array}{l}\text { Number of } \\
\text { layers }\end{array}$ & 3 & 3 & -- \\
\hline & Resolution & $176 \times 144$ & $88 \times 72$ & -- \\
\hline
\end{tabular}

Table 3. Available bandwidth and data sent for both receivers

Thanks to wireless clients/server applications, we demonstrate the efficiency of the proposed scalable system. Even if the performance of the proposed system still to be evaluated in harsh wireless environments, the proposed scalable system could be viewed as a valid step toward guaranteeing Quality of Service (QoS) for JPEG 2000 based multimedia transmission in heterogeneous Next Generation Wireless networks.

\section{References}

Taubman, D.S. \& Marcellin, M.W. (2001). JPEG 2000 Image Compression Fundamentals, Standards and Practice, In: Kluwer Academic Publishers, The Netherlands 2001

JPWL, (2005) JPEG 2000 part 11 Final Draft International Standard, ISO/IEC JTC 1/SC 29/WG 1 N3797

Dufaux, F. \& Nicholson, D. (2004). JPWL: JPEG 2000 for Wireless Applications, Proceeding of SPIE -- Volume 5558 - Applications of Digital Image Processing XXVII, Andrew G. Tescher, Editor, pp. 309-318, November 2004

Nicholson, D., Lamy-Bergot, C., Naturel, \& Poulliat, C. (2003). JPEG 2000 backward compatible error protection with Reed-Solomon codes. IEEE Transactions on Consumer Electronics, vol. 49, n. 4, pp.855-860, Nov. 2003

Agueh, M., Devaux, F.O. \& Diouris, J.F. (2007,a). A Wireless Motion JPEG 2000 video streaming scheme with a priori channel coding. Proceeding of 13th European Wireless 2007 (EW-2007), April 2007, Paris (France) 
Guo, Z., Nishikawa, Y., Omaki, R. Y., Onoye, T. \& Shirakawa, I. (2006). A Low-Complexity FEC Assignment Scheme for Motion JPEG 2000 over Wireless Network. IEEE Transactions on Consumer Electronics, Vol. 52, Issue 1, Feb. 2006 Page(s): 81 - 86

Agueh, M., Diouris, J.F., Diop, M., \& Devaux, F.O. (2007,b). Dynamic channel coding for efficient Motion JPEG 2000 streaming over MANET. Proceeding of Mobimedia2007, August 2007, Nafpaktos, Greece

Agueh, M., Diouris, J.F., Diop, M., Devaux, F.O., De Vleeschouwer, C. \& Macq,B. (2008). Optimal JPWL Forward Error Correction rate allocation for robust JPEG 2000 images and video streaming over Mobile Ad-hoc Networks. EURASIP Journal on Advances in Signal Proc., Spec. Issue wireless video, Vol. 2008, Article ID 192984, doi: 10.1155/2008/192984

Agueh, M., Ataman, S. \& Henoc, S. (2009, a). A low time-consuming smart FEC rate allocation scheme for robust wireless JPEG 2000 images and video transmission. Proceeding of Chinacom2009, 2009, Xi' an( China)

Agueh, M. \& Henoc, S. (2009, b). Optimal Layer-Based Unequal Error Protection for Robust JPEG 2000 Images and Video Transmission over Wireless Channels. Proceeding of MMEDIA2009, pp.104- 109, 2009 First International Conference on Advances in Multimedia, 2009

Schulzrinne, H. , Casner, S. , Frederick, R. \& Jacobson, V. (2003). RTP: A Transport Protocol for Real-Time Applications. STD 64, RFC 3550, July 2003.

Thomos, N., Boulgouris, N. V. \& Strintzis, M. G. (2004). Wireless transmission of images using JPEG 2000. Proceeding of ICIP'04, Singapore, October 2004.

Speedway video sequences have been generated by UCL.

Available: http://euterpe.tele.ucl.ac.be/WCAM/public/Speedway\%20Sequence/

Loss patterns acquired during the WCAM Annecy 2004 measurement campaigns IST-2003507204 WCAM, Wireless Cameras and Audio-Visual Seamless Networking. project website: http:/ /www.ist-wcam.org

Yee, J. R., \& Weldon E. J. (1995). Evaluation of the performance of error-correcting codes on a Gilbert channel. IEEE Transactions on Communications. vol. 43, n. 8, pp. 2316-2323, 1995

Descampe, A., De Vleeschouwer, C., Iregui, C., Macq, B. \& Marques, F. (2006). Pre-fetching and caching strategies for remote and interactive browsing of JPEG 2000 images. IEEE Transactions on Image Processing, vol 16, $\mathrm{n}^{\circ}$ 5, pp. 1339-1354, 2006

Wang, Z., Bovik, A. C. , Sheikh, H. R. \& Simoncelli, E. P. (2004). Image quality assessment: From error visibility to structural similarity. IEEE Transactions. on Image Processing., vol. 13,no. 4, pp 600-612, Apr. 2004

Fabrizio, F. \& Giuseppe, B. (2005). Backward-compatible interleaving technique for robust JPEG2000 wireless transmission Visual content processing and representation. Proceeding of 9th international workshop, VLBV 2005, Sardinia, Italy, September 15-16, 2005-VLBV 2005 No9, Sardinia, ITALIE (2005)

Li, M. \& Chang, C. (2009). A two-way available bandwidth estimation scheme for multimedia streaming networks adopting scalable video coding. Proceeding of IEEE Sarnoff Symposium, p1-p11, Princeton, USA, 2009. 
Devaux, F., Meessen, J., Parisot, C., Delaigle, J., Macq, B. \& De Vleeschouwer, C. (2007). A flexible video transmission system based on JPEG 2000 conditional replenishment with multiple reference. Proceeding of IEEE ICASSP 2007, Honolulu 2007, USA

Mairal, C. \& Agueh, M. (2010). Smooth and Scalable Wireless JPEG 2000 images and video streaming with dynamic Bandwidth Estimation. Proceeding Of the Second International conference on advances in Multimedia, June 2010, Athens, Greece

Johnsson, A. \& Björkman, M. (2008). On measuring available bandwidth in wireless networks. Proceeding of 33rd IEEE Conference on Local computer networks, 2008. Montreal, Canada 


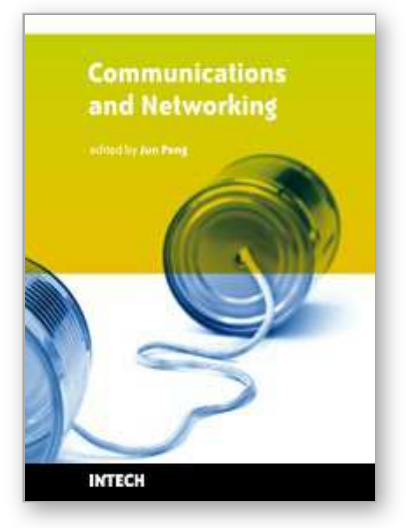

\section{Communications and Networking \\ Edited by Jun Peng}

ISBN 978-953-307-114-5

Hard cover, 434 pages

Publisher Sciyo

Published online 28, September, 2010

Published in print edition September, 2010

This book "Communications and Networking" focuses on the issues at the lowest two layers of communications and networking and provides recent research results on some of these issues. In particular, it first introduces recent research results on many important issues at the physical layer and data link layer of communications and networking and then briefly shows some results on some other important topics such as security and the application of wireless networks. In summary, this book covers a wide range of interesting topics of communications and networking. The introductions, data, and references in this book will help the readers know more abut this topic and help them explore this exciting and fast-evolving field.

\section{How to reference}

In order to correctly reference this scholarly work, feel free to copy and paste the following:

Max Agueh (2010). Wireless Multimedia Communications and Networking Based on JPEG 2000, Communications and Networking, Jun Peng (Ed.), ISBN: 978-953-307-114-5, InTech, Available from: http://www.intechopen.com/books/communications-and-networking/wireless-multimedia-communications-andnetworking-based-on-jpeg-2000

\section{INTECH}

open science | open minds

\section{InTech Europe}

University Campus STeP Ri

Slavka Krautzeka 83/A

51000 Rijeka, Croatia

Phone: +385 (51) 770447

Fax: +385 (51) 686166

www.intechopen.com

\section{InTech China}

Unit 405, Office Block, Hotel Equatorial Shanghai

No.65, Yan An Road (West), Shanghai, 200040, China

中国上海市延安西路65号上海国际贵都大饭店办公楼 405 单元

Phone: +86-21-62489820

Fax: $+86-21-62489821$ 
(C) 2010 The Author(s). Licensee IntechOpen. This chapter is distributed under the terms of the Creative Commons Attribution-NonCommercialShareAlike-3.0 License, which permits use, distribution and reproduction for non-commercial purposes, provided the original is properly cited and derivative works building on this content are distributed under the same license. 